\title{
A Case of Eosinophilia Associated With Olanzapine
}

\author{
Hidetaka Yamada $^{\mathrm{a}, \mathrm{b}, \mathrm{c}}$, Shigenari Ohmura ${ }^{\mathrm{a}}$, Naohisa Uchimura ${ }^{\mathrm{a}}$
}

\begin{abstract}
Olanzapine is an atypical antipsychotic medication that was originally developed for the treatment of schizophrenia. We report a case of eosinophilia possibly associated with olanzapine treatment for a recurrent manic episode. Although olanzapine is a potent agent for various psychiatric disorders, hematological complications should be taken into consideration as a possible adverse reaction.
\end{abstract}

Keywords: Olanzapine; Eosinophilia; Side effect

\section{Introduction}

Olanzapine is an atypical antipsychotic medication that was originally developed for the treatment of schizophrenia. It has also been shown to have broad efficacy in the treatment of bipolar disorder and manic episodes [1]. In addition to the commonly known adverse effects of this drug, including high blood sugar and/or weight gain, a few reports have indicated hematological toxicity as an adverse effect $[2,3]$. However, only one case report of eosinophilia has been published to date [4]. We report a case of eosinophilia possibly associated with olanzapine treatment for a recurrent manic episode.

\section{Case Report}

The patient, a 65-year-old woman, was admitted for dys-

\footnotetext{
Manuscript accepted for publication October 18, 2013

${ }^{a}$ Department of Neuropsychiatry, Kurume University School of Medicine, 67 Asahimachi, Kurume, Fukuoka, Japan

${ }^{b}$ Department of Psychiatry, Kurume University Medical Center, 155-1 Kokubumachi, Kurume, Fukuoka 839-0863, Japan

${ }^{\mathrm{c}}$ Corresponding author: Hidetaka Yamada, Department of Psychiatry, Kurume University Medical Center, 155-1 Kokubumachi, Kurume, Fukuoka 839-0863, Japan. Email: yamada_hidetaka@kurume-u.ac.jp

doi: http://dx.doi.org/10.4021/jmc1535w
}

pnea and fever and diagnosed with aspiration pneumonia. She was treated with antibiotics and a few days later, her symptoms improved. During the treatment, we were consulted for her decreased need to sleep, talkativeness, euphoric mood, excessive inquisitiveness, and hyperactivity. Her husband reported that she had been diagnosed with cyclothymic personality and developed hypomanic episode on occasion, although she had never been treated for those episodes. She was diagnosed with bipolar disorder and olanzapine treatment, $10 \mathrm{mg} /$ day, was started.

Before the beginning of olanzapine treatment, her white blood cell (WBC) count and eosinophil count were 5,120/L and $548 / \mathrm{L}$, respectively, making eosinophils $10.7 \%$ of the total WBC count. Four days after the induction of treatment, her WBC count had increased to 7,670/L, of which 2,000/L, or $26.0 \%$ of the total WBC count, was eosinophils. At this point, drugs possibly associated with eosinophilia such as antibiotics and NSAIDs were terminated and a parasitic infection and other causes of eosinophilia were excluded. Olanzapine treatment was discontinued in consultation with the internal medicine specialist. Two weeks after olanzapine cessation, the eosinophil counts had decreased to $12.0 \%$. In addition, the lymphocyte count had transiently decreased to $15 \%$ of the total WBC count, and had increased to the normal range as eosinophil counts declined.

\section{Discussion}

Blood eosinophilia, which is the presence of $>500 / \mathrm{L}$ of eosinophils, signifies either a cytokine-mediated reactive phenomenon (secondary) or an integral phenotype of an underlying hematological neoplasm (primary) [5]. Secondary eosinophilia is usually associated with parasitosis and allergic conditions including drug allergy [5]. Antibiotics or NSAIDs are often identified as a causative agent, and with regard to atypical antipsychotics, it is well established that clozapine can cause eosinophilia, which is often associated with organ-specific inflammatory processes [6]. One of the most distinctive findings of the present case is that the patient developed eosinophilia without leukocytosis. Eosinophilia is typically accompanied by leukocytosis and in the case re- 
ported by Mathias et al [4], the total WBC count increased up to $14.9 \times 10^{9} / \mathrm{L}$, half of which were eosinophils. Eosinophilia appears to be asymptomatic in general, yet eosinophil counts $>2,000 / \mathrm{L}$ can cause organ toxicity [6]. Although it is not known exactly why the total WBC count did not increase in our case, we should look for important diagnostic clues for allergic reactions to medications, including eosinophilia, by measuring differential leukocyte counts. In conclusion, olanzapine is a potent agent for various psychiatric disorders, including schizophrenia and manic disorder; however, impaired glucose tolerance and/or weight gain are common adverse effects, and hematological complications should be taken into consideration as a possible adverse reaction.

\section{References}

1. Lieberman DZ, Goodwin FK. Use of olanzapine in the treatment of bipolar I disorder. Expert Rev Neurother. 2004;4:759-767

2. Naumann R, Felber W, Heilemann H, Reuster T. Olanzapine-induced agranulocytosis. Lancet. 1999;354(9178):566-567.

3. Gajwani P, Tesar GE. Olanzapine-induced neutropenia. Psychosomatics. 2000;41:150-151.

4. Mathias S, Schaaf LW, Sonntag A. Eosinophilia associated with olanzapine. J Clin Psychiatry. 2002;63(3):246247.

5. Tefferi A, Patnaik MM, Pardanani A. Eosinophilia: secondary, clonal and idiopathic. Br J Haematol. 2006;133(5):468-492.

6. Roberts CE, Mortenson LY, Merrill DB, Rafizadeh N, Smith TE, Lieberman JA. Successful rechallenge with clozapine after eosinophilia. Am J Psychiatry. 2011;168(11):1147-1151. 